\title{
Prenatal Hand Expression of Breastmilk to Help Increase Breastfeeding Initiation and Exclusive Breastfeeding Rates
}

\section{Sally Chen ( $\sim$ chensallyb@gmail.com )}

Temple University Lewis Katz School of Medicine

Angela Liu

Temple University Lewis Katz School of Medicine

\section{Yukiko Washio}

Temple University Lewis Katz School of Medicine

\section{Gail Herrine}

Temple University Lewis Katz School of Medicine

\section{Research Article}

Keywords: breastfeeding, prenatal hand expression, colostrum expression, antenatal milk expression, pregnancy

Posted Date: February 10th, 2022

DOI: https://doi.org/10.21203/rs.3.rs-1333294/v1

License: (c) (i) This work is licensed under a Creative Commons Attribution 4.0 International License. Read Full License 


\section{Abstract}

\section{Background}

The objective of this study is to assess whether antenatal hand expression at 39 gestational weeks helps increase patient confidence and skill with hand expression and increases initiation and exclusive breastfeeding rates.

\section{Methods}

In this IRB-approved study, patients were consented at 34-39 weeks gestation and taught by an IBCLC to hand express, collect, and store colostrum and record output. Starting at 39 weeks gestation, participants were asked to practice hand expression 1-3 times/day until delivery. A postpartum survey assessed patient satisfaction with hand expression and opinions on breastfeeding self-efficacy. Chart review of postpartum or well-baby visit notes determined whether patients continued exclusively breastfeeding.

\section{$\underline{\text { Results }}$}

Of the 29 participants who delivered at Temple, 21/29 (72\%) were exclusively breastfeeding on discharge, and only $11 / 29(38 \%)$ reported exclusively breastfeeding at postpartum or well-baby visits. Barriers to the practice include maternal discomfort, low milk supply, and maternal and infant illness. Of the participants who completed the postpartum survey, $90 \%$ initiated breastfeeding and $72 \%$ reported hand expressing at home. They felt overall satisfied $(8.1 / 10, \pm 1.62)$ with prenatal hand expression and stated that it was helpful in breastfeeding initiation $(8.8 / 10, \pm 1.69)$.

\section{$\underline{\text { Conclusions }}$}

Prenatal hand expression starting as late as 39 gestational weeks still increased initiation and exclusive breastfeeding rates from $12 \%$ in the study period to $72 \%$ in the study group. Recognizing and addressing hand expression barriers during late pregnancy may help to further improve exclusive breastfeeding rates.

\section{Introduction}

The World Health Organization recommends that infants are exclusively breastfed until six months of age, and to continue breastfeeding after with complementary food. ${ }^{1}$ The benefits of breastfeeding for both mother and infant are well studied. For the child the benefits include but are not limited to decreased childhood ear and gastrointestinal infections, asthma, decreased rates of childhood obesity and diabetes. ${ }^{2-4}$ For the mother, benefits include decreased rates of hyperlipidemia, diabetes, cardiovascular disease, ovarian and breast cancer, decreased risk of postpartum depression, and increased postpartum weight loss. Also, for both mother and baby, breastfeeding increases bonding between mother and infant. $2-4$ 
Due to these maternal infant and benefits, worldwide orchestrated efforts have been made to increase awareness and to promote breastfeeding, such as the Baby Friendly Hospital Initiative by WHO and UNICEF, to help motivate facilities providing maternity and infant care by implementing the Ten Steps to Successful Breastfeeding, a summary of policies and procedures developed to support breastfeeding. ${ }^{5} \mathrm{~A}$ systematic review of 58 studies on maternity and newborn care published in 2016 demonstrated clearly that adherence to the Ten Steps impacts early initiation of breastfeeding immediately after birth, exclusive breastfeeding, and total duration of breastfeeding. ${ }^{6}$

Significant improvements in breastfeeding rates have been made in the past decade, and the push remains to increase exclusive breastfeeding rates. The WHO Global Targets 2025 include a target increase in the rate of exclusive breastfeeding in the first six months up to at least $50 \%{ }^{7}$ The $C D C$ healthy people 2020 objectives aimed to increase the proportion of infants ever breastfed to $81.9 \%$ and infants breastfed exclusive through six months to $25 \%{ }^{6}$

Despite an overall increase in breastfeeding initiation and exclusive breastfeeding rates in the nation, rates are variable between states, regions, and hospitals. Studies have also shown that women with lower levels of education, who are non-white, non-Hispanic, or of lower socioeconomic status face significant barriers and are less likely to meet their breastfeeding goals. ${ }^{8,9}$

Prenatal breastfeeding education helps improve breastfeeding rates and is a recommended strategy from the United States Preventive Services Task Force and the WHO/UNICEF. ${ }^{10}$ Most women make their breastfeeding decisions before conception and/or early in the pregnancy, which makes prenatal breastfeeding education and counseling vital. One study showed that breastfeeding education was only addressed at $29 \%$ of visits and for less than 40 seconds, which shows that breastfeeding may be inadequately addressed, especially during busy clinic visits. ${ }^{11,12}$ Healthcare worker-led intervention or an intensified healthcare provider training program for breastfeeding has been shown to significantly increase exclusive breastfeeding rates for six months and affect maternal knowledge about breastfeeding. ${ }^{13,14}$ Furthermore, in another study including low-income mothers who had Medicaid insurance or was uninsured, most participants agreed that educational breastfeeding interventions, such as on-demand videos, showing breastfeeding latch, positioning, benefits, neonatal behavior, and legal issues related to breastfeeding and work would have helped them exclusively breastfeed. ${ }^{15}$ Prenatal confidence in breastfeeding is one of the most significant predictors of breastfeeding duration. As such, women with low confidence in breastfeeding often feel that they have insufficient milk supply, start formula supplementation, and eventually stop breastfeeding. ${ }^{16}$ Prenatal classes, which addressed breastfeeding myths and concerns, may increase feelings of breastfeeding self-efficacy and showed greater rates of breastfeeding initiation and duration among low SES black women. ${ }^{16}$ Based on this evidence, ensuring that all pregnant mothers receive breastfeeding education can reasonably increase rates of breastfeeding. 
Hand expression is an effective technique for obtaining colostrum and it has been shown that in the postpartum period, mothers who hand expressed in the immediate postpartum period were more likely to be breastfeeding at two months (96.1\%) compared to mothers who used an electric pump $(72 \%) .{ }^{17,18} \mathrm{~A}$ recent 2018 cross sectional study of 688 women showed that $80.9 \%$ of surveyed women would consider antenatal breast expression if it was found to be helpful to prepare for breastfeeding. ${ }^{19}$ Several qualitative studies showed that confidence in breastfeeding increased with experiences of antenatal milk expression and that antenatal milk expression allowed women to store colostrum during pregnancy. ${ }^{20,21}$ A prospective study of 180 patients found that those who expressed breast milk daily after 37 weeks gestation found it easier to initiate breastfeeding and was found to have sufficient milk flowing within half an hour of initiation of breastfeeding (94.4\%) compared to patients in control group (70\%). ${ }^{22}$

However, the safety of antenatal hand expression has been highly debated as oxytocin release from antenatal nipple stimulation has been thought to cause adverse maternal and infant effects. Interestingly, a randomized controlled trial that examined antenatal hand expression starting at 36 weeks' gestation in women with diabetes in pregnancy demonstrated no maternal differences in labor onset, type of delivery, blood loss, or maternal hypoglycemia. ${ }^{23}$ Similarly, antenatal expression was not associated with worse infant outcomes, including admissions to the neonatal ICU, shorter mean gestation ages, lower birth weight, or lower Apgar scores. ${ }^{23}$ In fact, evidence suggests that antenatal hand expression was moderately associated with exclusive breastfeeding of newborns in the first twenty-four hours of life and during the initial hospital stay. ${ }^{23}$

Prior research has shown that prenatal breastfeeding education increases rates of breastfeeding and that many women are interested in antenatal breast expression to help prepare for breastfeeding. Current literature also suggests that antenatal hand expression can be safe in starting as early as 36 weeks' gestation, but our study examined if antenatal breast expression starting as late as 39 gestational weeks would still have an impact on maternal confidence in breastfeeding and help increase initiation and exclusive breastfeeding rates in our patient population.

\section{Methods}

Study Design and Setting: This study was reviewed and approved by the IRB at Temple University. Patients were recruited at 34-39 weeks gestation at the time of their prenatal visit at Temple Care Northeastern. All eligible patients were approached and given information regarding the study. Informed consent was obtained from study participants.

Participant Eligibility: The inclusion criteria were: (1) a pregnant female (2) between 18 years old or above, (3) were receiving prenatal care at the study site, (4) were able and willing to provide informed consent.

After electing to participate in the study, patients met with an International Board-Certified Lactation Consultant (IBCLC), who reviewed multimedia breastfeeding material to teach patients how to hand 
express colostrum. Patients were also given materials (medicine cups, syringes, Zipock bags) to collect and properly store colostrum for the duration of the study. Furthermore, patients were instructed to begin hand expression at home at 39 weeks gestation for one to three times each day, to document their milk output, to log their experiences, and to bring the frozen colostrum to the hospital upon delivery. Patients also received weekly phone calls or texts, depending on what the patient elected, to help encourage continued practice of hand expression and answer any questions.

After delivery, patients completed a postpartum survey, which included several questions rated on a 10point Likert scale. Patients were asked how satisfied they were with hand expression overall, whether they had ever hand expressed before this study, whether they practiced hand expression at home and how many times, whether they encountered any problems and what they were, if any, whether they thought practicing hand expression helped initiate breastfeeding more easily, and whether they would recommend hand expression to family or friends. To assess breastfeeding practices, the survey asked patients whether they had initiated breastfeeding and if they had ever breastfed in the past. Patients also rated their likelihood of continuing exclusively breastfeeding and what barriers they faced if they did not plan to continue exclusively breastfeed. Finally, retrospective chart review of postpartum and well-baby visit notes identified whether patients were exclusively breastfeeding in the hospital and if they continued that practice upon discharge.

\section{Results}

There was a total of 30 patients consented for this study with 27 of which who delivered at Temple University Hospital (TUH) and three of which who delivered at an outside hospital, one of which was lost to follow up. Half of the patients were 18-24 years old, and the other half was between 25-32 years. In this study population, $23 \%(7 / 30)$ of patients were enrolled in the study at $35-36$ weeks gestation, $70 \%$ $(21 / 30)$ of patients enrolled at 37-38 weeks, and only $6 \%(2 / 30)$ of patients enrolled at 39 weeks gestation. On average, patients had been pregnant three $( \pm 2.2)$ times, and six patients were primigravid. The mean gestational age at delivery was $39.8( \pm 0.52)$ weeks.

Breastfeeding: Of the 29 patients who completed the postpartum survey, as shown in Figure 1, 72\% $(21 / 29)$ were breastfeeding exclusively upon discharge from the hospital, but at postpartum or well-baby visits, only $38 \%(11 / 29)$ continued the practice. A total of $38 \%(11 / 29)$ of the patients had breastfed in the past, but with implementation of this study, 90\% (26/29) patients reported initiating breastfeeding in the hospital shortly after delivery. $55 \%$ (16/29) of patients planned to continue exclusively breastfeeding, but soon many patients faced multiple barriers to breastfeeding, which likely contributed to the decline in exclusive breastfeeding practices after leaving the hospital. As shown in Figure 2, the most common barrier was "low milk supply," cited by $39 \%$ (9/23) of patients, which led some mothers to supplement with formula and others to give up breastfeeding altogether, followed by other reasons including infant and maternal illness $(22 \% ; 5 / 23)$, poor latch $(17 \% ; 4 / 23)$, inconvenience $(17 \% ; 4 / 23)$, discomfort $(9 \%$; $2 / 23)$, and having returned to work $(9 \% ; 2 / 23)$. 
Hand Expression: Of the 29 patients who completed the postpartum survey, most were overall very satisfied with the practice of hand expression. Only $14 \%$ (4/29) of survey participants reported hand expressing in the past, as seen in Figure 3, but $72 \%$ (21/29) of the participants reported practicing and utilizing hand expression techniques at home in this study. None of the patients who initiated hand expression reported feeling contractions when practicing hand expression. Patients rated a satisfaction score of 8.1/10 ( \pm 1.62$)$ with hand expression overall, a score of 9.6/10 $( \pm 0.90)$ in their satisfaction toward the hand expression education provided by the IBCLC, and an 9.4/10 ( \pm 1.24$)$ likelihood of recommending hand expression to others. Most importantly, patients reported a score of 8.8/10 $( \pm 1.69)$ in how helpful hand expression was in initiating breastfeeding. However, $69 \%(9 / 13)$ of patients reported having a "low output" of milk and 31\% (4/13) reported "discomfort", as seen in Figure 4, which would lead them to frustration, impatience, and eventual discontinuation of hand expression.

\section{Discussion}

This study examined the preliminary effect of prenatal hand milk expression at 39 gestational weeks on exclusive breastfeeding rates at discharge and postpartum. In our study period from March 2020 to June 2021 , the average percent of exclusive breastfeeding for newborns discharged from the hospital was $12 \%$ (107/889). However, in this pilot study, we demonstrated that prenatal hand expression beginning at even 39 weeks gestation would increase breastfeeding initiation and exclusive breastfeeding rates in our patient population from $12 \%$ to $72 \%$, a drastic increase that could be attributed to several different factors.

Almost $90 \%$ of our obstetric patient population at the study site consists of Medicaid-insured patients. Current literature suggests that additional educational or multi-media information could impact breastfeeding rates in our patient population. ${ }^{13-15}$ Individual meetings with IBCLC and close follow-up from our research team as part of our study implementation likely contributed to the increase in exclusive breastfeeding rates compared to our general institutional obstetrics population. Overall, our patients were satisfied with the education provided to them at their prenatal visits.

Regarding the practice of hand expression, previous studies have shown that initiation of hand expression as early as 36 weeks gestation did not increase risk of maternal labor outcomes or infant factors, such as timing of labor onset, vaginal or cesarean delivery, amount of blood loss during delivery, neonatal ICU admission, or lower Apgar scores. ${ }^{23}$ While our study started hand expression at 39 gestational weeks, later than other studies, our patients still reported that hand expression education and behavior increased their comfort with breastfeeding and were likely to recommend hand expression to their family and friends. Furthermore, our patients did not report feeling contractions as a result of nipple stimulation, further adding to current literature that hand expression is likely safe in the antenatal period. Initiation of hand expression earlier than our study time frame can possibly contribute to greater comfort and confidence with expressing milk before delivery and further impact exclusive breastfeeding rates in the postpartum period. The current study findings demonstrated that hand expression starting as late as 
39 gestational weeks still contributes to maternal confidence in breastfeeding and helps to increase breastfeeding initiation and exclusive breastfeeding rates.

For those patients in our study who did initiate hand expression at home, effort and participation was also largely self-motivated, with some patients quickly becoming frustrated after a few attempts and other patients willing to try longer despite discomfort and low volume output. Participants in our study encountered several barriers to breastfeeding after discharge from the study site, including low supply, poor latch, inconvenience, work, discomfort, or maternal and infant illness. Pain with hand expression, perception of insufficient milk, concerns about maternal or infant health, or early in-hospital formula supplementation were all reasons why patients stopped breastfeeding earlier than desired. ${ }^{24}$ Future studies can consider more frequent check-ins with study personnel to encourage and motivate behavioral change in patients as well as provide additional opportunities for IBCLC to answer any questions that may arise throughout pregnancy and early postpartum periods. Moreover, future studies providing interventions to address socioeconomic barriers, such as helping mothers who must return to work or encouraging patients to avoid feeding formula just because of convenience, can help promote exclusive breastfeeding rates.

A few limitations in this study are acknowledged. First was unforeseen COVID pandemic, and a subsequent pause during recruitment period as modifications were made in protocol to accommodate changes from in office to remote operations including recruitment and consultations. As a result of COVID hospital visitor policy changes, participants and visitors were not allowed frequent entry or exit of hospital, so participants were given an insulated bag with ice packs to transport collected colostrum. The other limitation of this study is the relatively small sample size of 30 patients, with 29 patients who completed the postpartum survey.

In summary, this study adds to current literature by demonstrating that antenatal hand expression is helpful in initiating breastfeeding and increasing exclusive breastfeeding rates even when hand milk expression was practiced as late as 39 gestational weeks. It also examined barriers preventing patients from continuing exclusively breastfeeding and hand expressing. In providing patients with some clinical teaching by an IBCLC or other staff during prenatal visits, providers can encourage antenatal hand expression and increase breastfeeding initiation and exclusivity rates.

\section{Declarations}

Ethics approval and consent to participate

This study was approved by the Temple University Hospital Internal Review Board (\#26655), which takes into account ethical considerations. All participants provided written informed consent prior to enrollment in the study.

Consent for publication 
Not applicable.

Availability of data and materials

The data that support the findings of this study are available on request from the corresponding author [SC]. The data are not publicly available due to them containing information that could compromise research participant privacy/consent.

Competing interests

The authors declare that they have no competing interests.

Funding

This study was funded by a Keystone 10 Grant through the Pennsylvania Department of Public Health. The study was supported by a NIH grant. This work was supported by the National Institutes on Child Health and Human Development (R01HD094877).

Author's contributions

SC was responsible for data analysis, manuscript drafting, and critical revision. AL contributed to participant recruitment, data collection, manuscript drafting, and critical revision of this article. GH contributed to project conception and design, participant recruitment, data collection, manuscript drafting, and critical revision of the article. YW contributed to project conception and design and critical revision of the article.

Acknowledgements

Not applicable.

\section{References}

1. Fewtrell MS, Morgan JB, Duggan C, et al. Optimal duration of exclusive breastfeeding: What is the evidence to support current recommendations? In: American Journal of Clinical Nutrition. Vol 85. ; 2007. doi:10.1093/ajcn/85.2.635s

2. Salone LR, Vann WF, Dee DL. Breastfeeding: An overview of oral and general health benefits. Journal of the American Dental Association. 2013;144(2). doi:10.14219/jada.archive.2013.0093

3. Eidelman Al. Breastfeeding and the use of human milk: An analysis of the American academy of pediatrics 2012 breastfeeding policy statement. Breastfeeding Medicine. 2012;7(5). doi:10.1089/bfm.2012.0067

4. Lessen R, Kavanagh K. Position of the academy of nutrition and dietetics: Promoting and supporting breastfeeding. Journal of the Academy of Nutrition and Dietetics. 2015;115(3).

doi:10.1016/j.jand.2014.12.014 
5. Bass JL, Gartley T, Kleinman R. World Health Organization Baby-Friendly Hospital Initiative Guideline and 2018 Implementation Guidance. JAMA Pediatrics. 2019;173(1).

doi:10.1001/jamapediatrics.2018.3808

6. Pérez-Escamilla R, Martinez JL, Segura-Pérez S. Impact of the Baby-friendly Hospital Initiative on breastfeeding and child health outcomes: a systematic review. Maternal and Child Nutrition. 2016;12(3). doi:10.1111/mcn.12294

7. Gupta PM, Perrine CG, Chen J, Elam-Evans LD, Flores-Ayala R. Monitoring the World Health Organization Global Target 2025 for Exclusive Breastfeeding: Experience from the United States. Journal of Human Lactation. 2017;33(3). doi:10.1177/0890334417693210

8. Becker GE, Smith HA, Cooney F. Methods of milk expression for lactating women. Cochrane Database of Systematic Reviews. 2016;2016(9). doi:10.1002/14651858.CD006170.pub5

9. Sinha B, Chowdhury R, Sankar MJ, et al. Interventions to improve breastfeeding outcomes: A systematic review and meta-analysis. Acta Paediatrica, International Journal of Paediatrics. 2015;104. doi:10.1111/apa.13127

10. Chung M, Raman G, Trikalinos T, Lau J, Ip S. Interventions in primary care to promote breastfeeding: An evidence review for the U.S. preventive services task force. Annals of Internal Medicine. 2008;149(8). doi:10.7326/0003-4819-149-8-200810210-00009

11. Demirci JR, Bogen DL, Holland C, et al. Characteristics of breastfeeding discussions at the initial prenatal visit. Obstetrics and Gynecology. 2013;122(6). doi:10.1097/01.AOG.0000435453.93732.a6

12. Noel-Weiss J, Rupp A, Cragg B, Bassett V, Woodend AK. Randomized controlled trial to determine effects of prenatal breastfeeding workshop on maternal breastfeeding self-efficacy and breastfeeding duration. JOGNN - Journal of Obstetric, Gynecologic, and Neonatal Nursing. 2006;35(5). doi:10.1111/j.1552-6909.2006.00077.x

13. Kavle JA, Lacroix E, Dau H, Engmann C. Addressing barriers to exclusive breast-feeding in low- and middle-income countries: A systematic review and programmatic implications. Public Health Nutrition. 2017;20(17). doi:10.1017/S1368980017002531

14. Kim SK, Park S, Oh J, Kim J, Ahn S. Interventions promoting exclusive breastfeeding up to six months after birth: A systematic review and meta-analysis of randomized controlled trials. International Journal of Nursing Studies. 2018;80. doi:10.1016/j.ijnurstu.2018.01.004

15. Lewkowitz AK, Raghuraman N, López JD, MacOnes GA, Cahill AG. Infant Feeding Practices and Perceived Optimal Breastfeeding Interventions among Low-Income Women Delivering at a BabyFriendly Hospital. In: American Journal of Perinatology. Vol 36. ; 2019. doi:10.1055/s-0038-1676485

16. Kistin N, Benton D, Rao S, Sullivan M. Breast-feeding rates among black urban low-income women: Effect of prenatal education. Pediatrics. 1990;86(5).

17. Morton J. Is pumping out of hand? Why hand expression in the first 3 postpartum days is important. Lactation Matters, International Lactation Consultant Association.

18. Morrison B, Ludington-Hoe S, Anderson GC. Interruptions to breastfeeding dyads on postpartum day 1 in a University Hospital. JOGNN - Journal of Obstetric, Gynecologic, and Neonatal Nursing. 
2006;35(6). doi:10.1111/J.1552-6909.2006.00095.x

19. Fair FJ, Watson H, Gardner R, Soltani H. Women's perspectives on antenatal breast expression: A cross-sectional survey. Reproductive Health. 2018;15(1). doi:10.1186/s12978-018-0497-4

20. Demirci JR, Glasser M, Fichner J, Caplan E, Himes KP. "It gave me so much confidence”: First-time U.S. mothers' experiences with antenatal milk expression. Maternal and Child Nutrition. 2019;15(4). doi:10.1111/mcn.12824

21. Brisbane JM, Giglia RC. Experiences of expressing and storing colostrum antenatally: A qualitative study of mothers in regional Western Australia. Journal of Child Health Care. 2015;19(2). doi:10.1177/1367493513503586

22. Singh G, Chouhan R, Sidhu K. Effect of antenatal expression of breast milk at term in reducing breast feeding failures. Medical Journal Armed Forces India. 2009;65(2). doi:10.1016/S03771237(09)80125-1

23. Forster DA, Moorhead AM, Jacobs SE, et al. Advising women with diabetes in pregnancy to express breastmilk in late pregnancy (Diabetes and Antenatal Milk Expressing [DAME]): a multicentre, unblinded, randomised controlled trial. The Lancet. 2017;389(10085). doi:10.1016/S01406736(17)31373-9

24. Odom EC, Li R, Scanlon KS, Perrine CG, Grummer-Strawn L. Reasons for earlier than desired cessation of breastfeeding. Pediatrics. 2013;131(3). doi:10.1542/peds.2012-1295

\section{Figures}

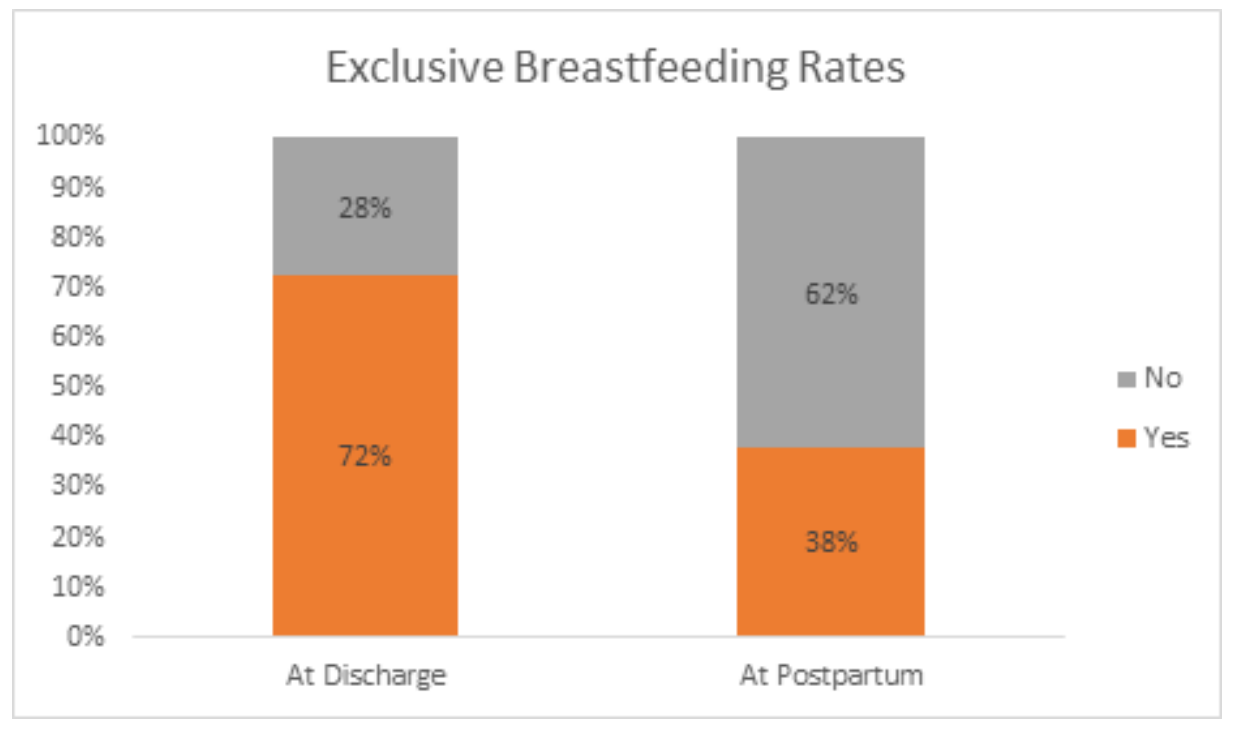

\section{Figure 1}

Figure legend not available with this version. 


\section{BARRIERS TO BREASTFEEDING EXCLUSIVELY}

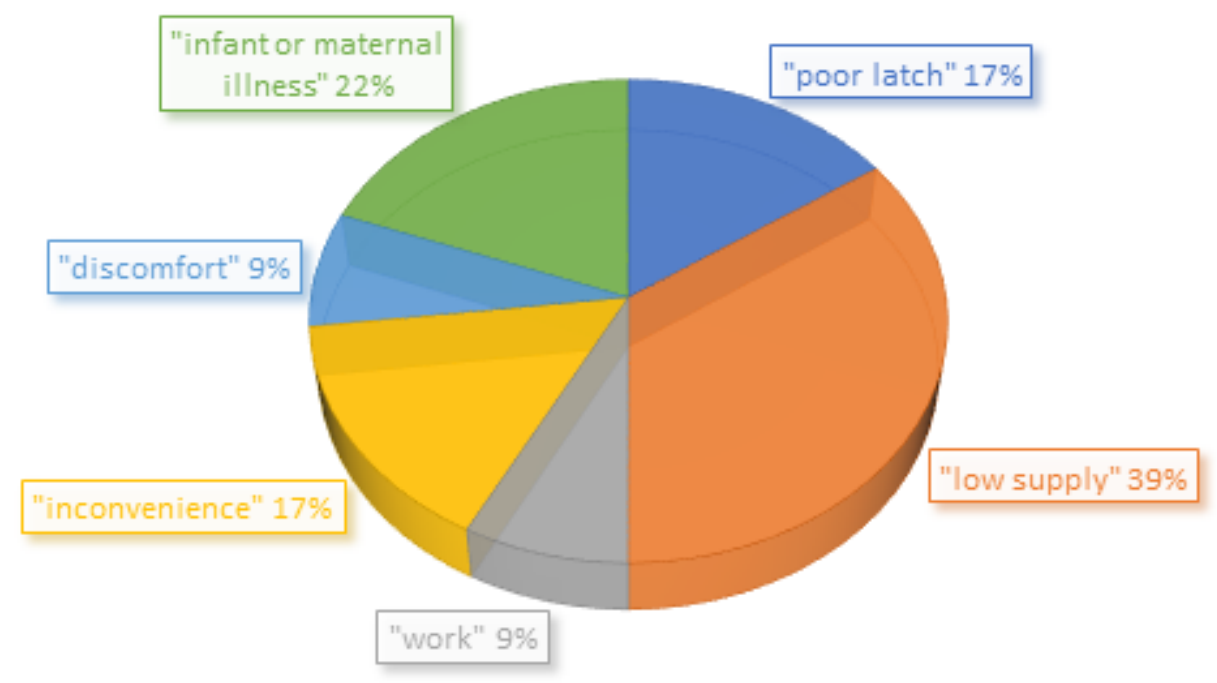

Figure 2

Figure legend not available with this version.

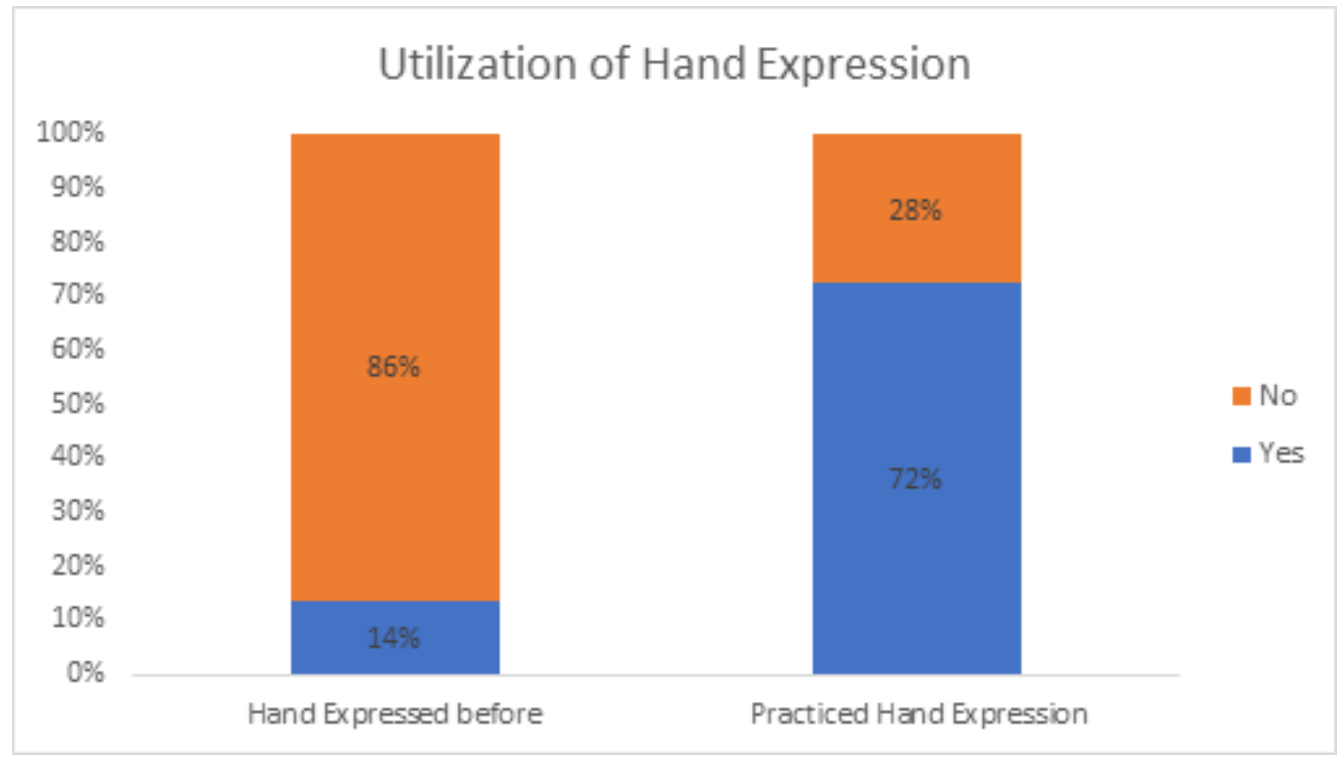

Figure 3

Figure legend not available with this version. 


\section{BARRIERS TO HAND EXPRESSION}

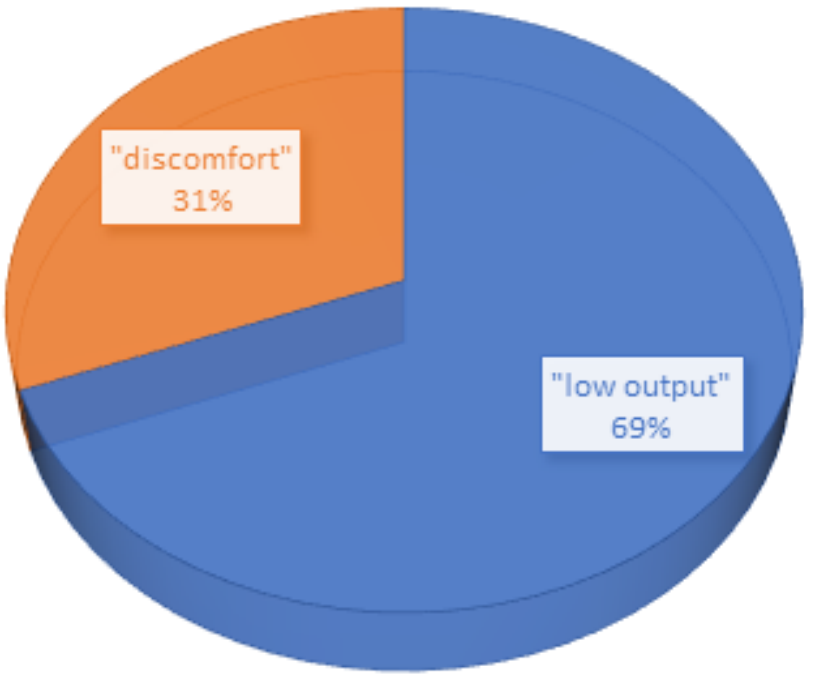

Figure 4

Figure legend not available with this version. 